\title{
Three Correlates of the Typological Frequency of Quantity-Insensitive Stress Systems
}

\author{
Max Bane and Jason Riggle \\ Department of Linguistics \\ University of Chicago \\ Chicago, IL 60637, USA \\ baneduchicago.edu, jriggle@uchicago.edu
}

\begin{abstract}
We examine the typology of quantityinsensitive (QI) stress systems and ask to what extent an existing optimality theoretic model of QI stress can predict the observed typological frequencies of stress patterns. We find three significant correlates of pattern attestation and frequency: the trigram entropy of a pattern, the degree to which it is "confusable" with other patterns predicted by the model, and the number of constraint rankings that specify the pattern.
\end{abstract}

\section{Introduction}

A remarkable characteristic of human language is that the typological distribution of many linguistic properties is extremely uneven. For example, Maddieson's (1984) survey of phonemic inventories finds that a total of 921 distinct sounds are used by a sample of 451 languages, yet the average language employs only about 30 of those. Furthermore, some sounds are so commonly attested as to be almost universal (e.g., $/ \mathrm{m} /, / \mathrm{k} /$ ), while others are vanishingly rare $(/ \mathbf{b} /, / \infty /)$. Heinz (2007) combines two previous typologies of accentual stress (Bailey, 1995; Gordon, 2002), and finds that among a sample of 306 languages with quantity-insensitive (QI) stress systems, 26 distinct stress patterns are found, ${ }^{1}$ while over $60 \%$ of the languages surveyed use one of just 3 of these patterns. If we begin to look at morphosyntactic or semantic properties, the combinatorics of

\footnotetext{
${ }^{1}$ These figures include only those quantity-insensitive stress patterns according to which there is exactly one possible assignment of stress per word length in syllables.
}

possible systems veritably explodes, leaving each attested language with an even smaller slice of the logical possibilities.

Most typological studies have attempted to give accounts of linguistic phenomena that simultaneously:

- predict as many attested languages or patterns as possible, and

- predict as few unattested languages or patterns as possible.

We will refer to this goal as the "inclusionexclusion" criterion of a linguistic model. Comparatively few attempts have been made to explain or predict the relative frequencies with which languages or patterns are observed to occur in crosslinguistic samples (though see Liljencrants and Lindblom 1972, de Boer 2000, Moreton to appear, and others for work proceeding in this direction).

This paper examines the typology of QI stress systems, as reported by Heinz (2007), and asks to what extent an existing optimality theoretic (Prince and Smolensky, 1993) model of QI stress, developed by Gordon (2002) to meet the inclusion-exclusion criterion, can predict the observed typological frequencies of stress patterns. Gordon's model predicts a total of 152 possible stress patterns, which, as far as we are aware, represent the current best attempt at satisfying the inclusion-exclusion criterion for QI stress, failing to generate only two attested stress patterns (unknown to Gordon at the time), and generating 128 unattested patterns. We show that Gordon's model can offer at least three novel, statistically significant predictors of which of the 152 generated patterns are actually attested, and of the 
cross-linguistic frequencies of the attested patterns. Namely:

i. Of the 152 stress patterns predicted by Gordon's model, the attested and frequent ones exhibit significantly lower trigram entropy than the unattested and infrequent,

ii. the length of forms, in syllables, that must be observed to uniquely identify a stress pattern is significantly lower for the attested patterns than for the unattested, and

iii. the number of constraint rankings in Gordon's model that are consistent with a stress pattern is a significant predictor both of which patterns are attested and of the relative frequencies of the attested patterns.

In what follows, Section 2 presents an overview of the basic theoretical background and empirical facts of quantity-insensitive stress that guide this study, including a review of Heinz's (2007) typology and a description of Gordon's (2002) OT model. Section 3 then introduces the three proposed correlates of attestedness and frequency that can be applied to Gordon's framework, together with statistical analyses of their significance as predictors. Finally, Section 4 offers a discussion of the interpretation of these findings, as well as some concluding remarks.

\section{Quantity-Insensitive Stress Patterns}

\subsection{Assumptions and Definitions}

We will follow Gordon (2002) and Heinz (2007) in taking a stress system to be any accentual system that satisfies "culminativity" in the sense of Prince (1983); that is, any accentual system in which there is always one most prominent accentual unit per accentual domain. In this case, we assume that the accentual unit is the syllable, and that the domain is the prosodic word. Thus, any given syllable of a word may bear primary, secondary, or no stress (we ignore the possibility of tertiary or other stress), but there must always be exactly one primary stressed syllable per word.

We further restrict our attention in this study to quantity-insensitive (QI) stress systems, which are those stress systems according to which the assignment of stresses to a word's syllables depends only

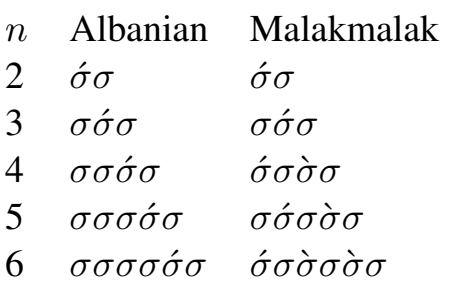

Table 1: The stress assignments of $n$-syllable words for $2 \leq n \leq 6$ in the QI stress patterns of Albanian and Malakmalak.

on the number of syllables present (a quantity assumed to be fixed when stress is assigned), and not on the segmental contents of the syllables. We will refer to "stress systems" and "stress patterns" interchangeably.

As two concrete examples of QI stress systems, consider those of Albanian (Chafe, 1977; also shared by many other languages) and Malakmalak (an Australian language; Birk, 1976). These patterns are illustrated in Table 1 for words of length two through six syllables. ${ }^{2}$ The former is a simple fixed system in which primary stress is always located on the penultimate syllable, while no other syllable bears stress. The latter is rather more complex, requiring stress on even numbered syllables from the right, the leftmost being primary. Crucially, neither system is sensitive to notions like syllabic weight, nor to any other properties of the syllables' contents.

Formally, one can consider a QI stress pattern up to length $n$ (in syllables), $P_{n}$, to be a set of strings over the alphabet $\Sigma=\{\sigma, \grave{\sigma}, \dot{\sigma}\}$ :

$$
P_{n}=\left\{w_{2}, \ldots, w_{n}\right\}
$$

where each $w_{i}$ encodes the locations of stress in a word of $i$ syllables, satisfying:

$$
\begin{aligned}
& \left|w_{i}\right|=i, \quad w_{i} \in \Sigma^{*}, \quad \text { and } \\
& w_{i} \text { contains } \sigma ́ \text { exactly once. }
\end{aligned}
$$

Thus for a given maximum number of syllables $n$, there are

$$
\prod_{i=2}^{n} i 2^{(i-1)}=n ! \cdot 2^{\frac{n(n-1)}{2}}
$$

\footnotetext{
${ }^{2}$ Here and throughout this paper, $\sigma$ refers to an unstressed syllable, $\grave{\sigma}$ indicates a syllable bearing secondary stress, and $\dot{\sigma}$ indicates primary stress.
} 


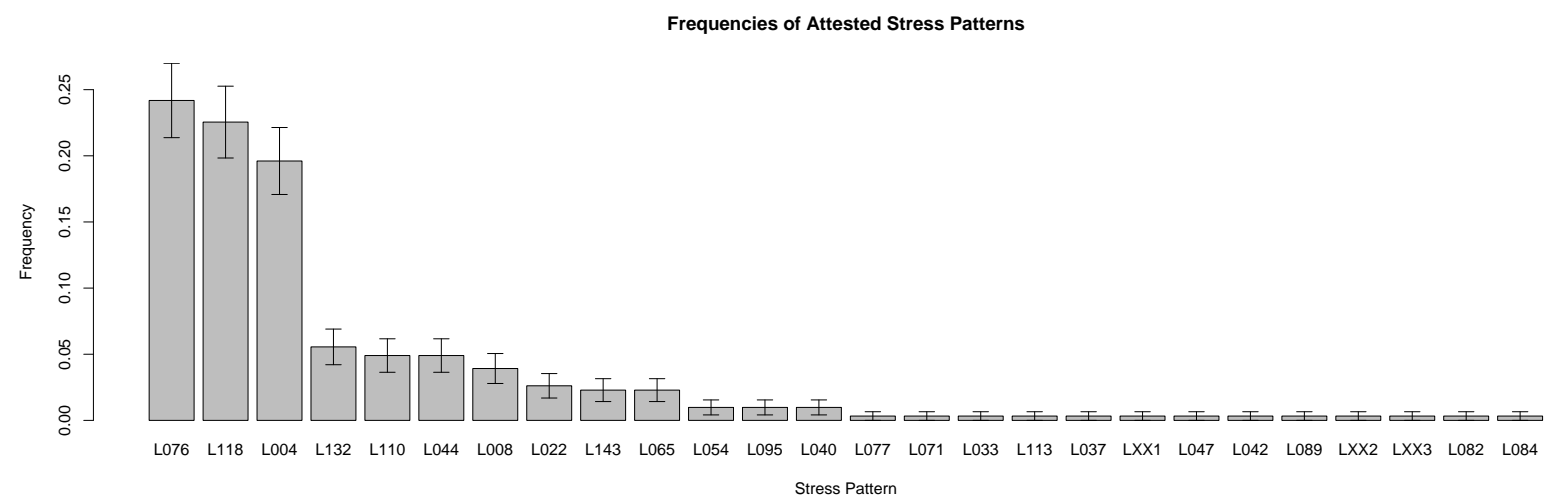

Figure 1: Frequency of attestation of each of the 26 distinct stress patterns. Error bars indicate standard Poisson sampling error.

logically possible QI stress patterns. We will follow Gordon (2002) by imposing a maximum word length of 8 syllables for purposes of distinguishing one stress pattern from another in the typology, and of determining the set of distinct patterns predicted by the model. We are therefore dealing with a universe of $8 ! 2^{28}=10,823,317,585,920$ theoretically possible stress systems.

\subsection{The Typology}

The typological data on which this study is based are due to Heinz (2007), who has made them freely available. ${ }^{3}$ This database is a combination of

- that from Bailey (1995), itself gathered from Halle and Vergnaud (1987) and Hayes (1995), and

- the collection put together by Gordon (2002) from previous surveys by Hyman (1977) and Hayes (1980), as well as from additional source grammars.

The combined database is intended to be fairly exhaustive, sampling a total of 422 genetically and geographically diverse languages with stress systems.

Of those 422 languages, 318 are identified as possessing quantity-insensitive stress, and we further confine our attention to the 306 of those with systems that uniquely determine the stress of each word as a function of syllable-count (i.e., with no optionality). We should note that it is possible for one lan-

\footnotetext{
${ }^{3}$ The typology is available as a MySQL database at http://www.ling.udel.edu/heinz/diss/
}

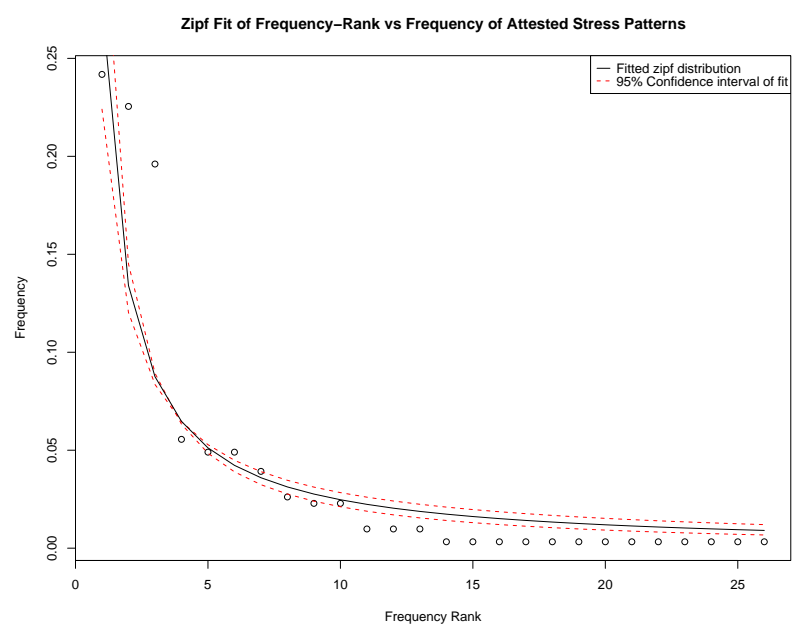

Figure 2: Regressed Zipf distribution of stress pattern frequencies; Zipf's exponent is found to be $1.05 \pm 0.15$ at $95 \%$ confidence.

guage to contribute more than one distinct stress pattern to our dataset, as in the case of Lenakel (Lynch, 1974), for instance, which employs one regular pattern for nouns and another for verbs and adjectives.

Between these 306 languages, we find a total of 26 distinct QI stress systems, which is quite a bit fewer than expected by chance, given the sample size and the 10.8 trillion a priori possible systems. Figure 1 shows the frequency with which each pattern is attested, arranged in decreasing order of frequency. The distribution of patterns is essentially Zipfian; a nonlinear regression of the frequencies against Zipf's law (using the Gauss-Newton method) achieves strong statistical significance ( $p<$ 0.001 ) and can account for $80.9 \%$ of the variance in 


\begin{tabular}{|c|c|}
\hline Constraint(s) & Penalizes... \\
\hline ALIGNEDGE & each edge of the word with no stress. \\
\hline $\operatorname{ALIGN}(\{\grave{\sigma}, \hat{\sigma}\}, \mathrm{L} / \mathrm{R})$ & $\begin{array}{l}\text { each (primary or secondary) stressed syllable for each other (stressed or un- } \\
\text { stressed) syllable between it and the left/right edge. }\end{array}$ \\
\hline $\operatorname{ALIGN}(\hat{\sigma}, \mathrm{L} / \mathrm{R})$ & $\begin{array}{l}\text { each primary stressed syllable for each secondary stressed syllable between it and } \\
\text { the left/right edge. }\end{array}$ \\
\hline NONFINALITY & the last syllable if it is stressed. \\
\hline *LAPSE & each adjacent pair of unstressed syllables. \\
\hline *CLASH & each adjacent pair of stressed syllables. \\
\hline *EXTLAPSE & each occurrence of three consecutive unstressed syllables. \\
\hline *LAPSELEFT/RIGHT & $\begin{array}{l}\text { the left/right-most syllable if more than one unstressed syllable separates it from } \\
\text { the left/right edge. }\end{array}$ \\
\hline *EXTLAPSERIGHT & $\begin{array}{l}\text { the right-most syllable if more than two unstressed syllables separate it from the } \\
\text { right edge. }\end{array}$ \\
\hline
\end{tabular}

Table 2: Gordon's (2002) constraint set.

frequency (Figure 2).

The top three most common patterns, together accounting for over $60 \%$ of the sampled languages, are all simple fixed primary stress systems: fixed final stress (24.2\% of systems), fixed initial stress $(22.5 \%$ of systems), and fixed penultimate stress (19.6\% of systems). It is possible that fixed primary systems may be somewhat overrepresented, as the descriptive sources can be expected to occasionally fail to report the presence of secondary stress; even so, the preponderance of such systems would seem to be substantial. The great majority of distinctly attested systems are quite rare, the median frequency being $0.65 \%$ of sampled languages. Some examples of cross-linguistically unlikely patterns include that of Georgian, with antepenultimate primary stress and initial secondary stress, and that of Içuã Tupi, which shows penultimate primary stress in words of four or fewer syllables, but antepenultimate stress in longer words.

There is some reason to believe that this sample is fairly representative of the whole population of QI stress patterns used by the world's languages. While it is true that the majority of sampled patterns are rare, it is by no means the case that the majority of sampled languages exhibit rare stress patterns. In fact, of the $N=306$ sampled languages, just $n_{1}=13$ of them present stress patterns that are attested only once. Thus, according to the commonly used Good-Turing estimate (a distributionfree method of estimating type frequencies in a pop- ulation from a sample of tokens; Good, 1953), we should expect to reserve approximately $\frac{n_{1}}{N}=4.3 \%$ of total probability-mass (or frequency-mass) for unseen stress patterns. In other words, we would be surprised to find that the actual population of languages contains much more than $\frac{N}{1-\frac{n_{1}}{N}}=27.15$ distinct patterns, i.e., about one more than found in this sample.

\subsection{Gordon's (2002) Model}

Gordon (2002) has developed an optimality theoretic model of QI stress with the goal of satisfying the inclusion-exclusion criterion on an earlier subset of Heinz's (2007) typology. The model is footless, consisting of twelve constraints stated in terms of a metrical grid, without reference to feet or other metrical groupings (or, equivalently, simply in terms of linear $\{\sigma, \grave{\sigma}, \dot{\sigma}\}$-sequences). The twelve constraints are summarized in Table 2.

In addition to these, Gordon's model implements a sort of "meta-constraint" on rankings: he assumes that one of the primary alignment constraints $\operatorname{ALIGN}(\sigma, L / R)$ is always lowest ranked, so that in any given tableau either $\operatorname{ALIGN}(\hat{\sigma}, \mathrm{L})$ or $\operatorname{Align}(\sigma, \mathrm{R})$ is "active," but never both. Formally, we take this to mean that the model specifies two Evals: an Eval-L with $\operatorname{Align}(\sigma, R)$ excluded from Con, and an Eval-R with $\operatorname{Align}(\sigma, L)$ excluded. The set of stress systems predicted by the whole model is then simply the union of the systems predicted by EVAL-L and by EVAL-R. This ranking 
restriction is meant to capture the probably universal generalization that primary stress always appears either to the left or right of the secondary stresses in a word, without vacillating from side to side for different word lengths. Gordon also assumes that candidate forms violating culminativity (i.e., forms without exactly one primary stressed syllable), are always excluded, either by some filter on the output of GEN or by an always highly ranked CULMINATE constraint against them. ${ }^{4}$

Gordon's model is capable of representing 2 . 11 ! = 79,833,600 QI stress grammars (11! rankings of the constraints associated with EVAL-L plus the 11! rankings for EVAL-R). We replicated Gordon's (2002) calculation of the factorial typology of distinct QI stress patterns that this grammar space predicts by implementing the constraints as finitestate transducers, ${ }^{5}$ composing the appropriate combinations of these to produce finite-state implementations of EVAL-L and EVAL-R, respectively (see Riggle, 2004), and iteratively constructing consistent subsets of the members of the cross-products of candidate forms for each word length (two through eight syllables). See Riggle et al (2007) and Prince (2002) for the mathematical and algorithmic details.

The factorial typology of stress systems that is yielded agrees with that reported by Gordon (2002). The model predicts a total of 152 distinct possible systems. All but two of the 26 systems attested in Heinz's (2007) database are among these. The two patterns that Gordon's model fails to generate are those of Bhojpuri (as described by Tiwari, 1960; Shukla, 1981), and Içuã Tupi (Abrahamson, 1968). Both of these patterns were unknown to Gordon at the time he proposed his model, and each is attested only once in the typology.

In addition to failing to generate two of the attested stress systems, Gordon's model also predicts

\footnotetext{
${ }^{4}$ We follow Gordon in remaining agnostic on this point, as the same set of possible stress patterns results from either implementation.

${ }^{5}$ The reader may notice that the $\operatorname{ALIGN}(\sigma, L / R)$ and $\operatorname{AligN}(\{\grave{\sigma}, \dot{\sigma}\}, \mathrm{L} / \mathrm{R})$ constraints (defined in Table 2$)$ involve a kind of counting that cannot generally be accomplished by finite-state transducers. This is perhaps a theoretically undesirable property of Gordon's model (see Heinz et al (2005) for such a critique), but in any case, this general problem does not affect us here, as we ignore the possibility of words any longer than eight syllables (following Gordon; see Section 2.1).
}

\section{Trigram Entropy}

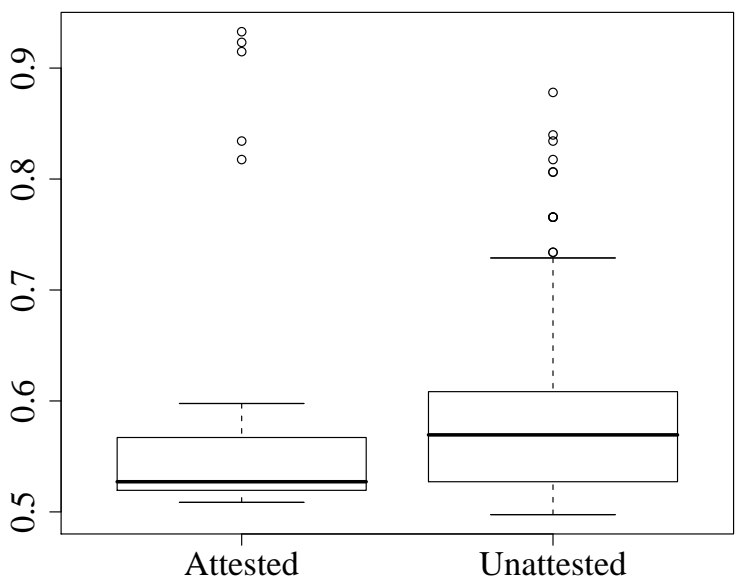

Figure 3: Trigram entropy (average bits per symbol) of attested versus unattested stress patterns; attested patterns have significantly lower entropy.

128 patterns that are unattested. Gordon (2002) argues that a certain amount of overgeneration is to be expected of any model, since the majority of distinct attested systems are extremely rare; thus failure to observe a pattern in a limited sample is not strong evidence that the pattern is impossible. The Good-Turing estimate of unseen patterns (Section 2.2 above), however, suggests that significantly less overgeneration may still be desired. Gordon also argues that the overgenerated patterns are not pathologically different from the sorts of patterns that we do see (though Section 3 below describes several statistically detectable differences). In any case, Gordon's model of QI stress is among the most explicitly formulated approaches currently available, and offers a comparatively "tight" fit to the typological data.

\section{Predicting Typological Frequency}

\section{1 $k$-gram Entropy}

A frequently offered and examined hypothesis is that, all else being equal, human communicative systems adhere to some principle of least effort (whether in terms of articulation or processing), preferring simple structures to complicated ones when additional complexity would afford no concomitant advantage in communicative efficiency or expressiveness. This line of reasoning suggests that typologically frequent properties should tend to exhibit 
(a)

\section{Frequency vs Trigram Entropy}

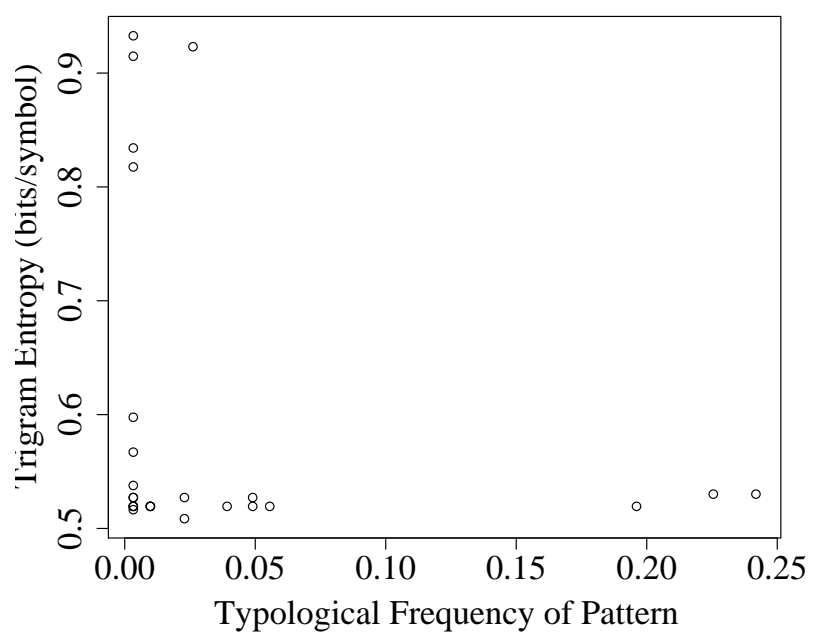

(b)

\section{Trigram Entropy}

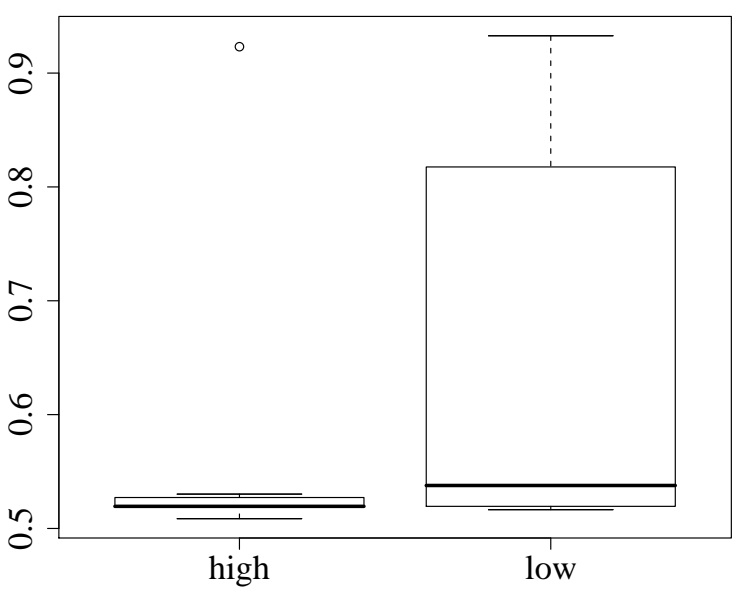

Figure 4: (a) typological frequency of attested stress patterns versus their trigram entropy, and (b) the trigram entropy of high-frequency (above median) patterns versus low-frequency (below median) patterns.

greater simplicity (according to some metric) than those that are rarer. One also expects, according to this hypothesis, that among the set of patterns predicted by a linguistic model such as Gordon's, the simpler ones should have a greater chance of attestation in typological samples. We find evidence consistent with both of these expectations in the case of QI stress systems, according to at least one information theoretic definition of complexity.

In order to calculate measures of complexity for each attested and predicted stress pattern, we construct bigram and trigram models of the transition probabilities between syllable types $(\sigma, \grave{\sigma}, \dot{\sigma})$ in forms of two through eight syllables for each pattern. That is, if each stress is taken to be a set of forms as in (1) (with $n=8$ in this case), satisfying (2), then across all forms (i.e., word-lengths) one can count the number of occurrences of each $k$-length sequence ( $k$-gram) of $\sigma, \grave{\sigma}, \hat{\sigma}$ and word boundaries to arrive at conditional probabilities for each syllable type (or a word boundary) given the previous $k-1$ syllables. With these probabilities one can then compute the Shannon entropy of the stress pattern as an index of its complexity; this is interpreted as the number of bits needed to describe the pattern (i.e., list its forms) under an efficient encoding, given the $k$-gram probability model. Stress patterns in which it is difficult to accurately predict the value of a syllable on the basis of the previous $k-1$ syllables will possess greater entropy, and thus be deemed more complex, than those in which such predictions can be made with greater accuracy.

We find that in the case of a bigram probability model $(k=2)$, the attested stress systems predicted by Gordon's model do not differ in entropy significantly ${ }^{6}$ from those that are unattested; we also find no significant correlation between bigram entropy and the typological frequency of attested systems.

Under a trigram probability model $(k=3)$, however, entropy is a significant predictor of both whether a system is attested, and if it is attested, of its frequency in the sample. Figure 3 gives boxplots comparing the distribution of trigram entropy for those systems predicted by Gordon's model (plus the two unpredicted systems) that are attested versus those that are unattested. The attested QI stress systems are significantly less entropic than the unattested, according to a two-sided Mann-Whitney $U$ test: $U=1196, p=0.021$ (if the two unpredicted patterns are excluded, then $U=923.5, p<0.01$ ). Among attested systems, trigram entropy appears to bear a nonlinear relationship to typological fre-

\footnotetext{
${ }^{6}$ Throughout this study, we adopt a $95 \%$ confidence standard of significance, i.e., $p<0.05$.
} 
quency (see Figure 4). A significant linear correlation does not exist, and the 13 attested patterns with greater than median frequency have only mildly significantly lower entropy than the 13 with less than median frequency (according to another two-sided $U$-test: $U=51.5, p=0.0856)$; if, however, the single high-frequency pattern with outlying entropy is excluded (the lone point indicated in Figure $4 b$ ), then the difference is more robustly significant: $U=39.5, p=0.0323$. Interestingly, the entropies of the above-median patterns are tightly constrained to a narrow band of values (variance 0.012 square bits/symbol), whereas the below-median patterns show much greater variation in their complexity (variance 0.028 square bits/symbol).

\subsection{Confusability Vectors}

The second metric we examine is motivated by considerations of learnability. Some QI stress patterns are very similar to each other in the sense that one must observe fairly long forms (i.e., forms with many syllables) in order to distinguish them from each other. For instance, in the case of Albanian and Malakmalak (Table 1 above), the two systems give identical stress assignments for words of two or three syllables; to tell them apart, one must compare words with four or more syllables. The degree of similarity, or "confusability" in this sense, between stress systems varies considerably for different pairs of languages. Assuming a tendency for short words to be encountered more frequently by language learners than long words, we might expect stress patterns that are easily identified at short word-lengths to be more faithfully acquired than those requiring longer observations for unambiguous identification. In particular, if we take the 152 patterns predicted by Gordon's model to constitute the set of possible QI stress systems, then we hypothesize that those patterns that stand out as unique at shorter lengths should be more typologically "stable": more likely to be attested, more frequently attested, or both.

To test this, we determine a confusability vector for each predicted pattern. This is simply a tuple of 7 integers in which the value of the $i$ th component indicates how many of the other 151 predicted patterns the given pattern agrees with on forms of two through $i+1$ syllables. For example, the confusabil-

\section{Syllable-Count for Unique Identification}

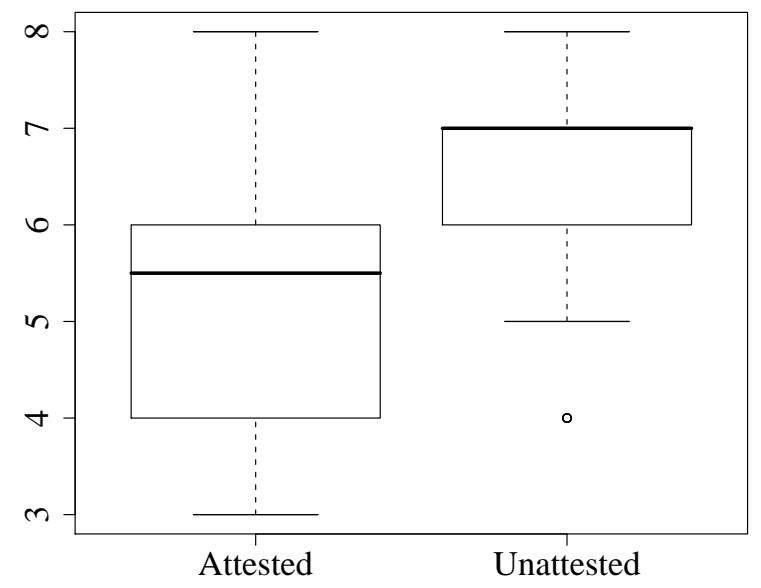

Figure 5: Attested stress patterns have significantly lower pivots than unattested ones.

ity vector of Albanian's (fixed penultimate primary; see Table 1) stress pattern is:

$$
\langle 101,39,10,0,0,0,0\rangle
$$

This means that for words of two syllables, this stress system agrees with 101 of the other predicted systems, for words of two through three syllables it agrees with 39, and for two through four syllables it agrees with 10. Once words of five or more syllables are included in the comparison, it is unique among the stress patterns predicted, confusable with none.

A confusability vector allows us to calculate two quantities for a given stress pattern: its confusability sum, which is just the sum of all the components of the vector, and a confusability pivot, which is the number $i$ such that the $(i-1)$ th component ${ }^{7}$ of the vector is the first component with value 0 . Thus the confusability sum of the fixed penultimate primary stress system is $101+39+10=150$, and its confusability pivot is 5 , indicating that it achieves uniqueness among Gordon's predicted systems at five syllables.

We find that those of the predicted systems that are typologically attested have very significantly lower confusability pivots than the unattested systems (see Figure 5; Mann-Whitney $U$-test: $U=$ 1005.5, $p<0.001$ ). One might wonder whether this is simply due to the fact that primary-only stress

\footnotetext{
${ }^{7}$ We count vector components beginning at 1 .
} 
systems are most likely to be attested, and that such systems are independently expected to have lower confusability pivots than those with secondary stress (indeed, a two-sided Mann-Whitney test indicates that the pivots of primary-only systems are significantly lower: $U=214, p<0.01)$. However, it appears that confusability pivots are in fact independently robust predictors of attestedness. When only the predicted patterns with secondary stress are considered, the pivots of the attested ones remain significantly lower than those of the unattested, albeit by a smaller margin $(U=846, p=0.027)$. Confusability sums, on the other hand, are not significant predictors of attestedness in either case.

Neither pivots nor sums alone correlate well with the typological frequency of attested systems, but together they can predict approximately $27 \%$ of the variance in frequencies; a multilinear regression of the form

$$
f(x)=\alpha+\beta s(x)+\gamma p(x),
$$

where $f(x), s(x)$, and $p(x)$ are the frequency, confusability sum, and pivot of pattern $x$, respectively, yields significant $(p<0.05)$ values for all coefficients $\left(R^{2}=0.271\right)$.

\subsection{Ranking Volume}

The two typological predictors discussed above (entropy and confusability) are only weakly "posttheoretical" in the sense that, while they depend on a set of predicted stress patterns according to some linguistic theory or model (such as Gordon's), they can be computed without reference to the particular form of the model. In contrast, the third and last correlate that we consider is entirely specified and motivated by the optimality theoretic form of Gordon's model.

We define the ranking volume, or $r$-volume, of a language generated by an optimality theoretic model as the number of total constraint orderings (i.e., grammars) that specify the language. Riggle (2008) describes a method of applying the logic of Prince's (2002) elementary ranking conditions to compute this quantity. Using this method, we find that the number of rankings of Gordon's constraints that are consistent with a stress pattern predicted by his model is a significant correlate of attestedness,

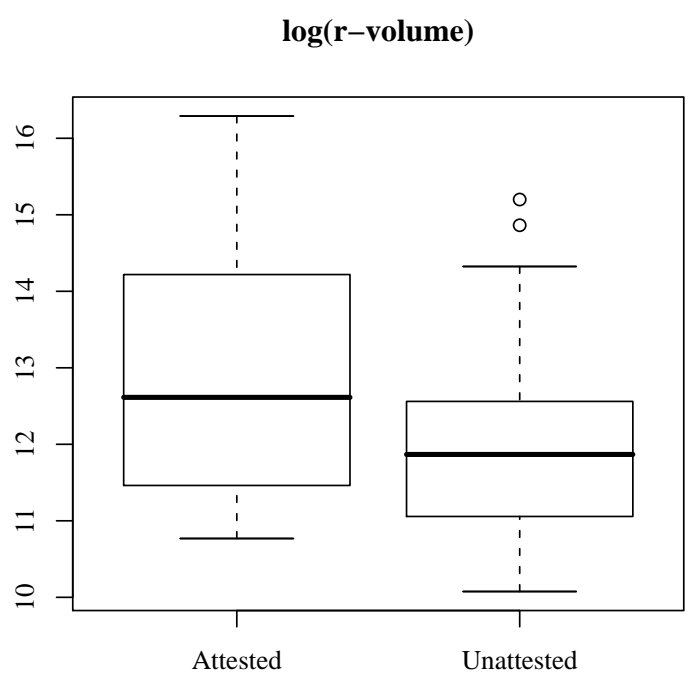

Figure 6: Of the predicted stress patterns, those that are attested are consistent with significantly more constraintrankings. The natural logarithms of $r$-volume are shown here for greater ease of comparison.

and if the pattern is attested, of its typological frequency. In the case of Gordon's model, with its ranking meta-constraint and bifurcated EvAL (as described in Section 2.3), the total $r$-volume of each pattern is actually the sum of two quantities: the pattern's $r$-volume under the 11 constraints corresponding to EVAL-L (which excludes $\operatorname{Align}(\hat{\sigma}, \mathrm{R})$ ), and its $r$-volume under the 11 constraints of EVAL-R (which conversely excludes $\operatorname{ALIGN}(\hat{\sigma}, \mathrm{R})$ ). Most of the predicted patterns are only generated by one of the Evals, but some can be specified by either constraint set, and thus will tend to be consistent with more rankings. It just so happens that Gordon's choice of constraints ensures that these doubly generated patterns are of precisely the same sort that are typologically most frequent: fixed primary stress systems. This appears to account for much of the predictive power of $r$-volume in this model.

The distribution of $r$-volume among the 152 predicted stress patterns is almost perfectly Zipfian. A nonlinear Gauss-Newton regression of $r$-volumes against Zipf's law finds a highly significant fit (with Zipf's exponent $=0.976 \pm 0.02, p<0.001$ ) that accounts for $96.8 \%$ of the variance. The attested patterns tend to have significantly greater $r$-volumes than those unattested; two-sided Mann-Whitney's $U=2113.5, p<0.01$ (see Figure 6). On aver- 


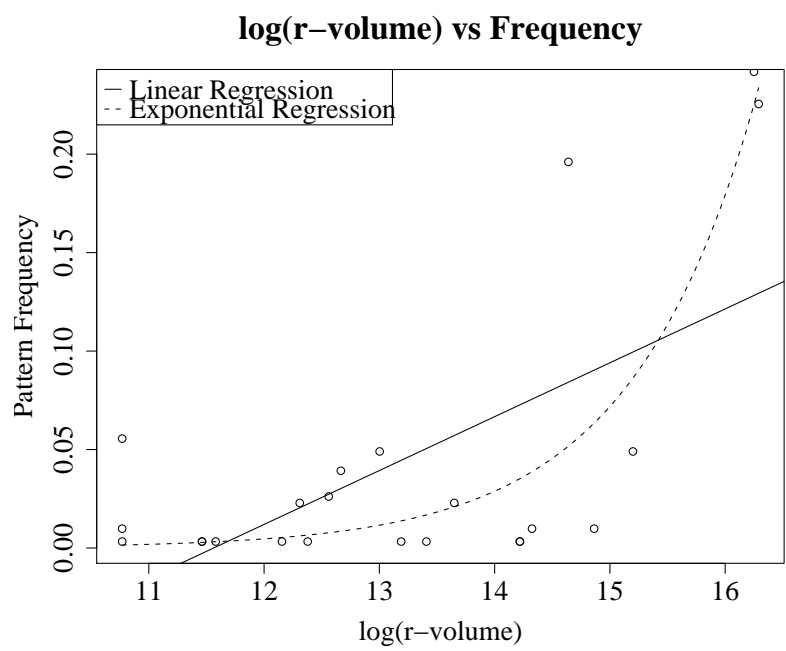

Figure 7: Linear and exponential regressions of typological frequency as a function of the natural logarithm of the pattern's $r$-volume.

age, the attested stress patterns are consistent with 1,586,437 rankings each, versus $299,118.1$ rankings for the unattested ones.

Furthermore, the frequency of attested patterns has a strong linear correlation with $r$-volume: $R^{2}=$ 0.7236, $p<0.001$. However, a linear relation is probably not appropriate, as a normal Q-Q plot of the residuals of the regression indicates an upper-quartile deviation from linearity, and Cook's distance metric indicates that several data-points exert disproportionate influence on the explained variance. Instead, typological frequency seems to be better modeled as a function of the logarithm of the $r$-volume; Figure 7 illustrates both a linear $\left(R^{2}=0.39, p<0.05\right)$ and exponential $\left(R^{2}=0.704, p<0.001\right)$ fit of frequencies to logtransformed $r$-volumes.

\section{Interpretation and Future Work}

The correlates of attestation and frequency reported here suggest novel ways that linguistic models might be used to make testable predictions about typology. Two of these correlates- $k$-gram entropy and confusability-are particularly general, their calculation requiring only the set of possible languages or patterns that a model can specify. It remains an interesting question whether these same quantities retain predictive power for other sorts of data and models than are considered here, and whether such correlations might fruitfully be incorporated into an evaluation metric for linguistic models.

The $r$-volume result motivates a particular line of further research on the nature of constraints in OT: how exactly the contents of a constraint set determine the distribution of $r$-volumes in the factorial typology. In addition, there are several other potentially relevant concepts in the literature, including Anttila's (1997, 2002, 2007) ranking-counting model of variation, Anttila and Andrus' (2006) “Torders" and Prince and Tesar's (1999) "restrictiveness measure," whose relations to $r$-volume merit examination. Our results for $r$-volume in this case also suggest that a fully generalized notion of parametric grammar volume may be worth investigating across different kinds of models and various typological phenomena.

Insofar as the three correlates' strength as typological predictors depends on the set of stress patterns generated by Gordon's model, their significance is consistent with the hypothesis that the model is useful and has some predictive power. Such statistical significance is rather surprising, since Gordon's model was developed primarily as an attempt to satisfy the inclusion-exclusion criterion, without any explicit eye toward the kinds of predictions that these correlates seem to suggest it can make. This is especially true of $r$-volume, as it is the correlate most tightly coupled to the OT particulars of Gordon's model. These findings motivate further research on the general relationship, if any, between the inclusion-exclusion predictions of a model (optimality theoretic or otherwise) and its frequency predictions according to the measures presented here. On the other hand, the entropy and confusability results suggest the intriguing possibility of discarding such a model altogether, and instead picking the attested stress systems (and their frequencies) directly from the large pool of logically possible ones, according to these measures and others like them.

\section{Acknowledgements}

We owe many thanks to Jeff Heinz for the typological data used in this study, and to Alan Yu, Morgan Sonderegger, and the anonymous reviewers of SIGMORPHON 2008 for insightful commentary. 


\section{References}

A. Abrahamson. 1968. Constrastive distribution of phoneme classes in Içuã Tupi. Anthropological Linguistics, 10(6):11-21.

Arto Anttila and Curtis Andrus. 2006. T-Orders. Manuscript, Stanford University.

Arto Anttila. 1997. Deriving variation from grammar. In Frans Hinskens, Roeland van Hout, and Leo Wetzels, editors, Variation, Change and Phonological Theory, pages 35-68. John Benjamins Press, Amsterdam/Philadelphia.

Arto Anttila. 2002. Variation and phonological theory. In Jack Chambers, Peter Trudgill, and Natalie Schilling-Estes, editors, Handbook of Language Variation and Change, pages 206-243. Blackwell, Malden, Mass.

Arto Anttila. 2007. Variation and optionality. In Paul de Lacy, editor, The Cambridge Handbook of Phonology. Cambridge University Press, Cambridge.

Todd Bailey. 1995. Nonmetrical Constraints on Stress. $\mathrm{Ph} . \mathrm{D}$. thesis, University of Minnesota.

D.B.W. Birk. 1976. The Malakmalak Language, Daly River (Western Arnhem Land). Australian National University, Canberra.

Bart de Boer. 2000. Self-organization in vowel systems. Journal of Phonetics, 28:441-465.

I.J. Good. 1953. The population frequencies of species and the estimation of population parameters. Biometrika, 40(3/4):237-264, December.

Matthew Gordon. 2002. A factorial typology of quantity-insensitive stress. Natural Language and Linguistic Theory, 20(3):491-552.

Morris Halle and Jean-Roger Vergnaud. 1987. An Essay on Stress. MIT Press, Cambridge, MA.

Bruce Hayes. 1980. A Metrical Theory of Stress Rules. Ph.D. thesis, MIT, Cambridge, MA.

Bruce Hayes. 1995. Metrical Stress Theory: Principles and Case Studies. University of Chicago Press, Chicago.

Jeffrey Heinz, Greg Kobele, and Jason Riggle. 2005. Exploring the typology of quantity-insensitive stress systems without gradient constraints. Handout, 2005 Annual Meeting of the Linguistic Society of America.

Jeffrey Nicholas Heinz. 2007. Inductive Learning of Phonotactic Patterns. Ph.D. thesis, UCLA.

Larry Hyman. 1977. On the nature of linguistic stress. In Larry Hyman, editor, Studies in Stress and Accent, pages 37-82. University of Southern California, Department of Linguistics, Los Angeles.

Johan Liljencrants and Bjorn Lindblom. 1972. Numerical simulation of vowel quality systems: The role of perceptual contrast. Language, 48(4):839-862.
John Lynch. 1974. Lenakel Phonology. Ph.D. thesis, University of Hawaii.

Ian Maddieson. 1984. Patterns of Sounds. Cambridge University Press, Cambridge.

Elliott Moreton. in press. Learning bias as a factor in phonological typology. In Charles Chang and Anna Havnie, editors, Proceedings of the 26th Meeting of the West Coast Conference on Formal Linguistics.

Alan Prince and Paul Smolensky. 1993. Optimality theory: Constraint interaction in generative grammar. Ms., Rutgers University and University of Colorado, Boulder.

Alan Prince and Bruce Tesar. 1999. Learning phonotactic distributions. Ms., ROA 535.

Alan Prince. 1983. Relating to the grid. Linguistic Inquiry, 14:19-100.

Alan Prince. 2002. Entailed ranking arguments. Rutgers Optimality Archive, ROA-500.

Jason Riggle, Max Bane, James Kirby, and Jeremy O'Brien. 2007. Efficiently computing OT typologies. In 2007 Annual Meeting of the Linguistic Society of America.

Jason Riggle. 2004. Generation, Recognition, and Learning in Finite State Optimality Theory. Ph.D. thesis, UCLA.

Jason Riggle. 2008. Counting rankings. Manuscript, University of Chicago. Draft available at http://hum.uchicago.edu/ jriggle/.

Shaligram Shukla. 1981. Bhojpuri Grammar. Georgetown University Press.

Udai Tiwari. 1960. The Origin and Development of Bhojpuri. Number 10 in Asiatic Society Monograph. Asiatic Society, Calcutta. 